\title{
Consulta de enfermagem ao portador de Hanseníase: proposta de um instrumento para aplicação do processo de enfermagem
}

\author{
Nursing consultation for Leprosy patients: proposal of an instrument for nursing process application \\ Consulta de enfermería al portador de la Lepra: propuesta de una herramienta para aplicación del proceso de enfermería
}

\section{Marli Teresinha Cassamassimo Duarte', Jairo Aparecido Ayres ${ }^{1}$, Janete Passuto Simonetti ${ }^{1}$}

'Universidade do Estado de São Paulo. Faculdade de Medicina de Botucatu, Departamento de Enfermagem. Botucatu, SP

Submissão: $30 / 8 / 2008$

Aprovação: 20/10/2008

\section{RESUMO}

Objetivou-se relatar a experiência da consulta de enfermagem junto aos portadores de hanseníase, realizada em unidade de atenção primária à saúde de uma Universidade do interior do Estado de São Paulo, bem como apresentar o instrumento utilizado. Essa atividade é realizada por dois enfermeiros, Que atuam por mais de duas décadas no programa, acumulando, assim, experiência no cuidado a esses indivíduos. Avaliam-se a eficiência dos instrumentos Quanto à forma e conteúdo, possibilitando o levantamento das reais necessidades de saúde, a partir de um olhar ampliado do processo saúde-doença. Com esta proposta, espera-se facilitar a atuação do enfermeiro na implementação das ações, visando o cuidado integral.

Descritores: Processo de Enfermagem; Hanseníase; Cuidados de enfermagem.

\section{ABSTRACT}

This study aimed at reporting the experience of nursing consultation for leprosy patients performed at a university primary health care unit in inner São Paulo state as well as at presenting the instrument used. This activity is performed by two nurses who have worked in the program for over two decades and have thus become experienced in providing care to this type of patient. The effectiveness of instruments is evaluated as concerns form and content, thus enabling the assessment of actual health care needs based on an expanded analysis of the health-disease process. With this proposal, it is expected that nurses' action will be facilitated in the implementation of procedures aiming at comprehensive care.

Descriptors: Nursing process; Leprosy; Nursing care.

\section{RESUMEN}

Se ha pretendido relatar la experiencia de la consulta de enfermería con los portadores de enfermedad de Hansen, realizada en una unidad de atención primaria a la salud de una universidad del interior del Estado de São Paulo, además de presentar el instrumento utilizado. Esa actividad es realizada por dos enfermeros que actúan desde hace más de dos décadas en el programa y acumulan, así, experiencia en el cuidado a esos individuos. Se evalúa la eficiencia de los instrumentos en cuanto a la forma y contenido, lo Que posibilita la averiguación de las reales necesidades de salud con una mirada ampliada del proceso salud-enfermedad. Con esta propuesta, se espera facilitar la actuación del enfermero en la implementación de las acciones con caras al cuidado integral.

Descriptores: Proceso de Enfermería; Lepra; Atención de enfermería. 


\section{INTRODUÇÃO}

O objetivo deste trabalho foi relatar a experiência da consulta de enfermagem junto aos portadores de hanseníase, realizada em unidade de atenção primária à saúde, bem como apresentar o instrumento utilizado. Inicia-se com uma breve retrospectiva dos aspectos epidemiológicos, clínicos e históricos da hanseníase e discussão conceitual sobre necessidades de saúde e consulta de enfermagem, com a finalidade de contextualizar a experiência, Que se desenvolve em uma Unidade Básica de Saúde, do interior do Estado de São Paulo.

A hanseníase é uma doença crônica, infecto-contagiosa, Que tem como características peculiares a evolução lenta e as manifestações dermatoneurológicas, determinadas pela reposta imune do individuo acometido ${ }^{(1,2)}$.

As alterações neurológicas ocorrem por lesões nos troncos nervosos periféricos, causadas tanto pela ação direta do bacilo, Quanto pelos estados reacionais. Manifestam-se por meio de dor e/ou espessamento neural, diminuição ou perda de sensibilidade e/ou da força motora, nas áreas com inervação afetada e, Quando não diagnosticadas e tratadas precoce e adequadamente, levam a incapacidades $^{(1,3)}$. Os principais nervos acometidos são: cubital, mediano, fibular, tibial posterior e facial, podendo levar a anestesias e paralisias, com potencial para o desenvolvimento de deformidades incapacitantes, como garra de artelhos, absorções ósseas, lagoftalmo, dentre outras ${ }^{(1)}$.

Essas incapacidades e deformidades podem acarretar problemas para o doente como diminuição da capacidade de trabalho, limitação da vida social e problemas psicológicos, sendo responsáveis, também, pelo estigma e preconceito contra a enfermidade ${ }^{(4)}$.

No Brasil, em 2006, foram notificados 47.612 casos novos, dos Quais $8 \%$ ocorreram em menores de 15 anos, aproximadamente $6 \%$ apresentavam grau II de incapacidade e $53 \%$ eram multibacilares $(\mathrm{MB})^{(5)}$.

Dessa forma, a alta detecção de casos no Brasil associada ao seu potencial incapacitante, torna a hanseníase um importante problema de saúde pública.

A hanseníase parece ser uma das doenças mais antigas Que acometem o homem. Ao longo da história, as medidas tomadas para enfrentar o problema da hanseníase tiveram por base o isolamento do doente, Que não se revelou capaz de controlar a endemia e contribuiu, sobremaneira, para aumentar o medo e o estigma associados à doença. $\mathrm{O}$ isolamento compulsório, como recomendação para o controle da doença, persistiu até o início da década de 60 , tendo sido oficialmente abolido em $1962^{(6)}$.

Até recentemente a hanseníase não tinha tratamento específico. As sulfonas foram descobertas na década de 1940, trazendo a possibilidade do tratamento ambulatorial; na década de 1960, a clofazimina vem corroborar com a recomendação do tratamento ambulatorial e, apenas nos anos 70, com o surgimento da Rifampicina, com seu poder bactericida, se tem o conceito de cura. A partir de 1989 a polieuimioterapia foi introduzida no Brasil, se constituindo em instrumento fundamental na eliminação da hanseníase ${ }^{(7)}$.

O tratamento da hanseníase é fundamental na estratégia de controle da doença, enQuanto problema de saúde pública. Ele tem o objetivo de interromper a transmissão da doença, Quebrando a cadeia epidemiológica, como também, prevenir incapacidades físicas, promover a cura e a reabilitação física e social do doente.

Dentre as diretrizes básicas Que objetivam a redução da morbimortalidade por hanseníase no âmbito do Sistema Único de Saúde (SUS), destaca-se a atenção integral ao portador de hanseníase Que deve ser garantida pela hierarQuização de serviços e pelo cuidado por equipe multiprofissional $^{(2)}$.

A consulta de enfermagem deve ser executada de forma sistemática para os doentes e seus comunicantes ${ }^{(2)}$.

\section{A consulta de Enfermagem e o Conceito de Clínica Ampliada}

A partir da década de 20, a entrevista realizada pelo enfermeiro foi considerada como uma atividade precursora da consulta de enfermagem. Em 1968, surgiu com a denominação consulta de enfermagem entendida como atividade dirigida prioritariamente ao grupo materno-infantil. Posteriormente, foi abrangendo outros grupos inscritos nos programas de saúde Que visavam ao controle da tuberculose, da própria hanseníase e de outras doenças crônicodegenerativas, como a diabetes e a hipertensão arterial ${ }^{(8)}$.

A regulamentação da consulta de enfermagem, "exercida de fato e não de direito nos serviços de saúde" ${ }^{\text {(8) }}$ em nível nacional, dá-se pela Lei no 7498/86 e pelo Decreto no 94406/87 Que, em seu artigo $11^{\circ}$, a legitima e a determina como modalidade de prestação de assistência direta ao cliente, atividade privativa do enfermeiro. A Resolução COFEN-159/93, artigo I ${ }^{\circ}$ torna a consulta de enfermagem obrigatória no desenvolvimento da assistência de enfermagem em todos os níveis de atenção à saúde, seja em instituição pública ou privada.

A consulta de enfermagem é proposta como exemplo de aplicação individual do Processo de Enfermagem ${ }^{(9)}$, constituindose este em dinâmica das ações sistematizadas e inter-relacionadas Que visam o cuidado ${ }^{(10)}$. Contempla os seguintes passos do processo de enfermagem: histórico de enfermagem (inclui a entrevista e o exame físico), diagnóstico de enfermagem, prescrição de enfermagem e implementação da assistência e evolução de enfermagem. Dessa forma, ela tem por princípio o conhecimento das necessidades de saúde para a proposição da prescrição e implementação da assistência de enfermagem ${ }^{(10)}$.

O reconhecimento das necessidades de saúde são elementos potenciais no auxílio dos trabalhadores da saúde para "se fazer uma melhor escuta das pessoas que buscam cuidados de saúde", tomando suas necessidades como centro de suas intervenções e prática $^{(11)}$.

Alguns autores propõem Que os serviços de saúde se organizem de modo a satisfazer não só as necessidades conhecidas, mas Que criem espaços Que possibilitem à clientela expor suas necessidades relacionadas à saúde ${ }^{(12)}$.

Ressalta-se, assim, a importância de se resgatar a prática clínica Que não decodifica apenas Questões biopsíQuicas, mas Que reconheça valores de vida, condições sociais, formas de enfrentamento de problemas, adotando-se posturas Que possibilitem conhecer, não apenas sinais e sintomas biológicos do indivíduo, mas também a sua maneira de "andar na vida"(13).

A clínica ampliada baseia-se na reconstrução da prática clínica, agregando além dos agravos, os problemas de saúde e as situações Que ampliam o risco ou vulnerabilidade das pessoas. Entende-se 
por essa ampliação a valorização da subjetividade do indivíduo. Além disso, considera-se essencial a ampliação também do objetivo ou finalidade do trabalho clínico, Que além de buscar a produção de saúde, por meios curativos, preventivos e de reabilitação, deverá contribuir para a ampliação da autonomia dos usuários ${ }^{(14)}$.

O grau de autonomia se eleva com o aumento da capacidade dos usuários de compreenderem e atuarem sobre si mesmos e sobre o mundo da vida, e assim pode ser medido pela capacidade do autocuidado, da compreensão do processo saúde/doença e do uso do poder de estabelecimento de compromisso e contrato com outros $^{(14)}$.

Portanto, cabe ressaltar Que a consulta de enfermagem é uma atividade primordial na assistência, pois estabelece uma interação terapêutica do indivíduo e o profissional da saúde, o Que possibilita o reconhecimento das condições de vida Que determinarão os perfis de saúde e doença ${ }^{(13)}$, além de direcionar a prática profissional para a independência, autonomia e Qualidade de vida dos indivíduos ${ }^{(15)}$. A agregação de elementos da história de vida de cada pessoa, do contexto social e cultural poderá propiciar a identificação de fatores de risco e de proteção. Desta forma, as ações propostas não se restringirão à terapêutica medicamentosa, mas com a valorização do poder terapêutico da escuta e da palavra, da educação em saúde e do apoio psicossocial ${ }^{(14)}$.

A complexa busca no reconhecimento das necessidades de saúde, tem por objetivo a integralidade do cuidado. Ainda Que se reconheça Que a integralidade do cuidado só poderá ser alcançada com o trabalho em eQuipe, com o entrelaçamento dos seus múltiplos saberes e práticas, a consulta de enfermagem poderá promover a "integralidade focalizada"(11).

Estudos referendam a importância da consulta de enfermagem na busca do atendimento integral, com vistas à maior resolutividade dos problemas de saúde dos usuários dos serviços ${ }^{(13,16)}$.

Tendo em vista os aspectos sócio-culturais e biológicos Que envolvem a hanseníase, bem como a importância da autonomia e autocuidado, ressalta-se o potencial da consulta de enfermagem como estratégia de cuidado ao seu portador.

\section{RELATO DA EXPERIÊNCIA}

Este relato de experiência deriva-se da pesquisa "Consulta de Enfermagem ao portador de Hanseníase: proposta de uma modalidade de assistência em Enfermagem em Atenção Primária à Saúde" Que segue os princípios da resolução 196/96, Protocolo número $27 \mathrm{I} / 2003$, no Que se refere à pesquisa com seres humanos, sendo realizada após esclarecimento dos sujeitos em relação aos objetivos da mesma e assinatura do Termo de Consentimento Livre e Esclarecido.

As consultas de enfermagem são realizadas pelos autores, no Centro de Saúde Escola (CSE), unidade de atenção básica da Faculdade de Medicina de Botucatu. O Programa de Assistência ao Portador de Hanseníase é referência para os municípios da microregião de Botucatu, Departamento Regional de Saúde DRS VI, Bauru. Visa à assistência integral ao portador de hanseníase e seus comunicantes, bem como, ações de prevenção na comunidade e minimização do estigma associado à doença. Dentre estas, divulgação nos meios de comunicação de massa de aspectos da doença, com destaQue para a cura com o tratamento; programa de educação permanente em hanseníase, dirigidos aos profissionais da região e realização periódica de busca ativa de casos na comunidade, em parceria com equipes de outras unidades de saúde e/ou instituições.

Essa experiência vem se concretizando ao longo de mais de duas décadas, e em meados de 2003 foram elaborados e implementados instrumentos de consulta de enfermagem denominados: Consulta de Enfermagem ao Cliente Portador de Hanseníase - Caso Novo (anexo I) e Consulta de Enfermagem ao Cliente Portador de Hanseníase - Seguimento, objetivando o atendimento sistematizado.

Os instrumentos têm por base o processo de enfermagem proposto por Horta ${ }^{(5)}$, porém ao invés das seis fases, foram adaptados para Quatro fases distribuídas em três partes: Histórico de Enfermagem; Levantamento das Necessidades de Saúde e Prescrição de Enfermagem e Evolução de Enfermagem.

No Instrumento Consulta de Enfermagem ao Cliente Portador de Hanseníase - Caso Novo (Anexo I) o Histórico de Enfermagem compreende: dados de identificação, aspectos do ambiente, Queixas, antecedentes pessoais e familiares, hábitos de vida, aspectos sócioeconômicos e rede de apoio, conhecimentos sobre a hanseníase, reações frente ao diagnóstico, aspectos do tratamento atual, exame físico geral e específico para avaliação do grau de incapacidades dos olhos, mãos e pés, conforme padronização do Ministério da Saúde ${ }^{(4)}$. Este instrumento é utilizado na primeira consulta de enfermagem ao portador de hanseníase.

O instrumento Consulta de Enfermagem ao Cliente Portador de Hanseníase - Seguimento, é utilizado nas consultas de retorno do cliente, contribuindo, também, para monitorar as intervenções propostas inicialmente. Diferencia-se do utilizado na primeira consulta apenas no Histórico de Enfermagem, Que é mais sintético, tendo em vista Que várias informações já foram obtidas anteriormente. Neste instrumento, o Histórico de Enfermagem atenta-se para as Queixas atuais; adesão ao tratamento; atividades rotineiras; atividades laborais e sociais; assim como, dificuldades encontradas na realização das propostas terapêuticas pactuadas; exame físico geral e dermatoneurológico. A parte do instrumento que contempla o levantamento das necessidades de saúde, prescrição e evolução de enfermagem é idêntica em ambos os instrumentos.

Para maior praticidade, baseados na literatura e experiência dos autores, formulou-se um check-list Que contempla os principais problemas/necessidades de saúde e prescrições de enfermagem, voltadas para aspectos específicos da hanseníase, reservando-se espaço nos impressos para incorporação de outras necessidades individuais.

À medida que foram sendo utilizados, os instrumentos sofreram algumas adeQuações, com a finalidade de contemplar a proposta de abrangência dos aspectos que envolvem a doença e o indivíduo comprometido.

As consultas de enfermagem são realizadas bimensalmente, intercalando-se com as consultas médicas ou com maior freqüência, conforme as necessidades da implementação da assistência de enfermagem e/ou necessidades dos próprios pacientes. São atendidos clientes tanto durante o tratamento polieuimioterápico, Quanto no acompanhamento destes pós-alta, uma vez Que, em ambos os momentos requerem cuidados de enfermagem sistematizados. 
O tempo médio da consulta de "Caso Novo" varia de uma a duas horas, dependendo de fatores como: idade, escolaridade, incapacidades instaladas e necessidades manifestadas pelos clientes. Em alguns casos, faz-se necessário realizar a avaliação de incapacidades em outro momento, por conta de cansaço ou restrição na disponibilidade de tempo do cliente, complementando-se, assim, a prescrição de enfermagem ao término da avaliação. As consultas de seguimento duram, em média, 30 minutos.

Em ambas as modalidades de consulta, após contemplar as etapas do processo proposto, o plano de cuidados é pactuado com o cliente, enfocando-o como responsável pelo autocuidado. Para isso, discutem-se as situações de risco e vulnerabilidades, como por exemplo, presença de áreas anestésicas, presença de reações, situações de discriminação, como também, aspectos protetores, como rede de apoio.

Destaca-se Que o instrumento de Consulta de Enfermagem ao Cliente Portador de Hanseníase - Seguimento, não será apresentado e discutido neste trabalho, visto Que o mesmo continua sendo aplicado aos clientes Que já foram atendidos, com intuito de avaliar os resultados das prescrições de enfermagem, sendo este, foco de estudos posteriores.

\section{CONSIDERAÇÕES FINAIS}

A prática da consulta de enfermagem permitiu a reflexão sobre seu potencial enquanto estratégia de abordagem integral do cliente, considerando outros fatores envolvidos no processo saúdedoença, como os psicossociais, culturais e familiares dos indivíduos, sem, no entanto, deixar de evidenciar aqueles específicos da hanseníase.

Embora se reconheça Que nossa apreensão da realidade seja sempre parcial e a partir de um ponto de vista, reside-se aí, o desafio da enfermagem, que é "Quebrar este olhar cristalizado dentro de nós, para abrirmos possibilidades de apreensão do que é saúde-doença e do Que pode ser alternativa de solução para os problemas Que se nos apresentam "(17).

A experiência na utilização dos instrumentos de consulta de enfermagem propostos tem mostrado a eficiência destes Quanto à forma e conteúdo, uma vez que possibilitam um olhar ampliado do processo saúde-doença, facilitando-se, assim, a atuação do enfermeiro na abordagem integral do cliente. A prescrição de enfermagem pactuada constitui-se em estratégia de desenvolvimento da autonomia dos clientes, podendo favorecer o autocuidado. No entanto, a identificação dos resultados das recomendações propostas na prescrição de enfermagem, encontrase em fase de análise para posterior divulgação.

Embora, se reconheça a pertinência do uso do instrumento para se aprimorar a consulta de enfermagem, ressalta-se a importância da Qualificação do enfermeiro para a atenção ao portador de hanseníase.

\section{REFERÊNCIAS}

I. Scollard DM, Adams LB, Gillis TP, Krahenbuhl IL, Truman, Williams DL. The continuing challenges of leprosy. Clin Microbial Rev 2006; 49(2): 338-81.

2. Ministério da Saúde (BR). Secretaria de Políticas de Saúde. Departamento de Atenção Básica. Guia para o controle da Hanseníase. Brasília: Ministério da Saúde; 2002.

3. Goulart IMB, Penna GO, Cunha G. Imunopatologia da Hanseníase: a complexidade dos mecanismos da resposta imune do hospedeiro do Mycobacterium leprae. Rev Soc Bras Med Trop 2002; 35(4): 365-75.

4. Ministério da Saúde (BR). Fundação Nacional de Saúde. Manual de prevenção de Incapacidades. Brasília: Ministério da Saúde; 1997.

5. World Health Organization [homepage on the Internet]. Situation report: leprosy in the Americas, 2007. Geneva (CH): WHO; 2008 [cited 2008 jul 29]. Available from: URL: http:// www.paho.org/English/AD/DPC/CD/lep-sit-reg-2007.pdf

6. Opromolla DV. História da Hanseníase. In: Noções de Hansenologia. Bauru: Centro de Estudos "Dr. Reynaldo Quagliato" Hospital "Lauro de Souza Lima”; 1981. p. I-9.

7. Secretaria de Estado da Saúde de São Paulo (SP). PCH Programa de Controle da Hanseníase. Campanha Estadual de Combate à Hanseníase. São Paulo: CVE - Centro de Vigilância Epidemiológica; 2006.

8. Adami NP, Franco LHRO, Brêtas ACP, Ransan LMO, Pereira AL. Características básicas que diferenciam a consulta de enfermagem da consulta médica. Acta Paul Enferm 1989; 2(1): 9-13.
9. Nogueira MIC. O processo de enfermagem em enfermagem comunitária: uma proposta. Rev Paul Enferm 1993; 3(1): 15-20.

10. Horta WA. Processo de Enfermagem. São Paulo: EPU; 1979.

11. Cecílio LCO. As necessidades de saúde como conceito estruturante na luta pela integralidade e eqüidade na atenção em saúde. In: Pinheiro R, Mattos RA, organizadores. Os sentidos da integralidade na atenção e no cuidado à saúde. Rio de laneiro: IMS/UERJ/ ABRASCO; 2001. p. I 13-26.

12. Schraiber LB, Mendes-Gonçalves RB. Necessidades de saúde na atenção primária. In: Schraiber LB, Nemes MIB, MendesGonçalves RB, organizadores. Saúde do adulto: programas de ações na unidade básica. São Paulo: Hucitec; 2000. p. 29-47.

13. Fracolli LA, Bertazolli MR. A abordagem do processo saúdedoença das famílias e do coletivo. In: Manual de Enfermagem. Brasília: Instituto para o Desenvolvimento da Saúde, Universidade de São Paulo, Ministério da Saúde; 2001 . p. 4-8.

14. Campos GWS, Amaral MA. A clínica ampliada e compartilhada, a gestão democrática e redes de atenção como referenciais teórico-operacionais para a reforma do hospital. Cienc Saúde Coletiva 2007; 12(4): 849-59.

15. Faeda A, Leon CGRMP. Assistência de enfermagem a um paciente portador de Diabetes Mellitus. Rev Bras Enferm 2006; 59(6): 818-21.

16. Vieira VB, Patine FS, Paschoal VDA, Brandão VZ. Sistematização da assistência de enfermagem em um ambulatório de hanseníase: estudo de caso. ArQ Cienc Saúde 2004; I I (2): 2- 10.

17. Matumoto S, Mishima SM, Pinto IC. Saúde Coletiva: um desafio para enfermagem. Cad Saúde Pública 200 I; 17(I): 233-4I. 


\section{Agradecimento:}

Agradecemos a Noêmia Macedo, Ludmila Braga, Ana Fávia e Neíse Milanesi que compõem a equipe de atenção ao portador de Hanseníase do CSE e a Profa. Dra. Heloísa W. Berti pelo incentivo a apresentação deste trabalho.

\section{ANEXO \\ CONSULTA DE ENFERMAGEM AO CLIENTE PORTADOR DE HANSENÍASE - CASO NOVO}

HISTÓRICO DE ENFERMAGEM
I. DADOS PESSOAIS
Nome:
Sexo: ( ) Estado civil:
Anos completos de estudo:
Data do Diagnóstico:
Situação do Tratamento:

\section{AMBIENTE}

2.I. Condições de habitação: ( ) alvenaria ( ) madeira ( ) material alternativo No de cômodos:___Relação morador/Quarto:

\begin{tabular}{|c|c|c|}
\hline Iluminação: ( ) ótima & ( ) boa & ( ) regular ( ) ruim \\
\hline ( ) ótima & ( ) boa & ( ) regular ( ) ruim \\
\hline Água: & ( ) poço & ( ) mina \\
\hline ( ) rede & ( ) céu aberto & ( ) fossa \\
\hline
\end{tabular}

Destino dos resíduos sólidos: ( ) coleta pública $3 \times$ ou $+/$ semana ( ) coleta pública 2 x / semana

( ) coleta pública I x/ semana( ) Queima

( ) outro:

Iluminação elétrica: ( ) sim ( ) não

Há alguma condição no seu ambiente Que o desagrada?

2.2. Condições de trabalho: Onde trabalha?

Ocupação: Como realiza o trabalho?

Como é o ambiente de trabalho? (relação espaço físico/trabalhador; iluminação; aeração. presença de ruídos). Há alguma condição no ambiente Que o incomoda?

\section{QUEIXAS}

\section{ANTECEDENTES PESSOAIS E FAMILIARES}

Apresenta alguma outra doença?

HÁ ( ) DM ( ) Cardiopatias ( ) Dislepidemias ( ) Neoplasias ( ) Outras

Medicamentos em uso:

Apresenta alguma doença na família? HA ( ) DM ( ) Neoplasias ( ) Outras:

\section{INVESTIGAÇÃO DOS APARELHOS:}

Sistema nervoso: cefaléia ( ); tonturas ( ); desmaios ( ); outros

Sistema cárdio-respiratório: falta de ar ( ); palpitações ( ); dor pré-cordial ( ); outros

Sistema Digestivo: náuseas ( ); vômitos ( ); Queimação ( ); dor ( ); outros

Eliminação urinária: freeüência

Eliminação intestinal: freQüência:

G P A C MAC:

cia:

Prevenção de próstata ; sintomas: consistência:

Padrão sexual: vida sexual ativa ( ) sim não ( ) Alterações após o aparecimento da doença?

Imunização: dT: esquema completo? sim ( ) última dose ___ não ( )

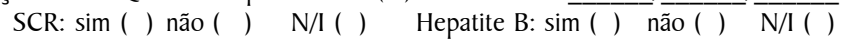

\section{HÁBITOS DE VIDA}

6.1. Alimentação habitual: refeições/dia: principais alimentos:
6.2. Tabaco:
6.3 Álcool:
( ) $\operatorname{Sim}($ ) $\operatorname{sim}$
) Sim ( ) Não Quantidade/dia:
6.4 Outras drogas:
6.5. Ativ. Física:
( ) Não
( ) Sim ( ) Não Qual:
6.6 Ativ. de lazer (freqüência):
6.7 Sono:
( ) Calmo ( ) Agitado
( ) Sim ( ) Não
Freqüência:
Repouso/dia:

7. ASPECTOS SÓCIO-ECONÔMICOS/COMUNICANTES/ REDE DE APOIO

7.1 Casa própria ( ) Casa cedida ( ) Casa alugada ( ) Outra:

7.2 Quem reside/residiu nos últimos 5 anos na sua casa? 


\section{Nome}

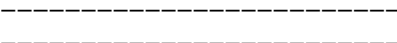

Renda familiar aproximada:

7.3 Como é sua relação com a família? Tem atritos constantes com alguém?

7.4 Como é sua relação no trabalho? Tem atritos constantes com alguém?

7.5 Quem você procura Quando precisa de ajuda? Com Quem você pode contar?

\section{CONHECIMENTO SOBRE A HANSENÍASE}

8. I O(a) $\mathrm{Sr}$ (a). sabe o nome da doença para a Qual está fazendo este tratamento?

$8.2 \mathrm{O}$ Que o(a) $\mathrm{Sr}(\mathrm{a})$. sabe sobre ela?

8.3 Teve contato com outras pessoas portadoras de Hanseníase/Que tratavam de alguma doença de pele? Quem?

\section{REAÇÕES FRENTE AO DIAGNÓSTICO}

9.I Como o $\operatorname{Sr}($ a) recebeu o diagnóstico de hanseníase?

$9.2 \mathrm{O}$ Que significa para o(a) $\mathrm{Sr}(\mathrm{a})$ ter/ ter tido hanseníase?

10. SOBRE O TRATAMENTO ATUAL (aderência/dificuldades)

10.1 Tem tido dificuldades em comparecer às consultas médicas/Enfermagem/coleta de exames?

( ) Sim ( ) Não Qual?

10.2 Tem tido alguma dificuldade com os remédios da Hanseníase?

I 0.3 Como tem tomado?

10.4 Em Que situações deixa de tomar?

EXAME FÍSICO

Peso:__ Kg Estatura: $\mathrm{Kg}$ Estatura: $\quad \mathrm{cm} \quad \mathrm{IMC}=$ $\mathrm{PA} \uparrow_{\rightarrow}:$ Braço ( ) Cardíaco:
Pulmonar:

Outros achados:

N. Radial D: Abdominal:

ãos:

N. Mediano D:

N. Mediano E:

N. Fibu

N. Fibular E:

$N$ Tibial Posterior D:

N. Tibial posterior E:

O registro da avaliação de incapacidades de Mãos/Pés/Olhos/Nariz é realizado nos impressos propostos pelo Ministério da Saúde ${ }^{(4)}$. 
II LEVANTAMENTO DAS NECESSIDADES DE SAÚDE E PRESCRIÇÃO DE ENFERMAGEM

\begin{tabular}{|c|c|c|}
\hline PROBLEMAS/NECESSIDADES & PRESCRIÇÃO DE ENFERMAGEM & DATA \\
\hline ( ) Déficit de conhecimento sobre a doença & ( ) Orientação aspectos gerais da doença e do tratamento & \\
\hline ( ) Ressecamento da pele & $\begin{array}{l}\text { ( ) Orientação para Hidratação e lubrificação da pele e } \\
\text { massagem }\end{array}$ & \\
\hline $\begin{array}{l}\text { ( ) Sinais e sintomas sugestivos de reações: } \\
\text { Dor espontânea : } \\
\text { Dor à palpação: } \\
\text { Espessamento: } \\
\text { Tinel }+ \text { : } \\
\text { ENH }\end{array}$ & $\begin{array}{l}\text { Orientação Quanto: } \\
\text { ( ) Repouso de membros } \\
\text { ( ) Medicação prescrita e modo de usar } \\
\text { ( ) Realização de talas }\end{array}$ & \\
\hline ( ) Sinais e sintomas relacionados ao aparelho ocular & ( ) Orientação para lubrificação ocular artificial & \\
\hline ( ) Dieta e/ou hábitos alimentares inadequados & ( ) Orientação dieta/hábitos alimentares saudáveis & \\
\hline ( ) Não comparecimento de comunicantes p/ exame e vacina BCG & $\begin{array}{l}\text { ( ) Orientação da importância do comparecimento dos } \\
\text { comunicantes } \\
\text { ( ) Realizar convocação de comunicantes }\end{array}$ & \\
\hline ( ) Incapacidade/ Deformidade instalada & $\begin{array}{l}\text { Orientação Quanto: } \\
\text { ( ) A prevenção de infecções } \\
\text { ( ) Cuidados com olhos/mãos/pés anestésicos } \\
\text { ( ) Adaptação de instrumentos de trabalho e vida diária } \\
\text { ( ) Exercícios de fortalecimento da musculatura }\end{array}$ & \\
\hline ( ) Calosidades em mãos e/ou pés & ( ) Orientação lixar e retirar calosidades & \\
\hline ( ) Maceração e/ou fissuras em interdígitos & ( ) Orientação secagem interdígitos e cuidado com calçados & \\
\hline ( ) Nível pressórico elevado & ( ) Realização de mapa pressórico & \\
\hline \multirow[t]{4}{*}{$\begin{array}{l}\text { ( ) Sinais e sintomas sugestivos de transtornos emocionais e/ou } \\
\text { sofrimento mental }\end{array}$} & $\begin{array}{l}\text { Orientações Quanto: } \\
\text { ( ) Importância da adesão ao tratamento } \\
\text { ( ) Proteção solar }\end{array}$ & \\
\hline & ( )Encaminhamentos: & \\
\hline & ( )Ajudar: & \\
\hline & ( ) Supervisionar: & \\
\hline
\end{tabular}

III EVOLUÇÃO DE ENFERMAGEM

NOME: COREN: 